\title{
Genetic characterization and molecular survey of Babesia bovis, Babesia bigemina and Babesia ovata in cattle, dairy cattle and yaks in China
}

Qingli Niu, Zhijie Liu', Peifa Yu', Jifei Yang', Mirza Omar Abdallah', Guiquan Guan', Guangyuan Liu', Jianxun Luo ${ }^{1^{*}}$ and Hong Yin ${ }^{1,2^{*}}$

\begin{abstract}
Background: Babesiosis is an important haemoparasitic disease, caused by the infection and subsequent intraerythrocytic multiplication of protozoa of the genus Babesia that impacts the livestock industry and animal health. The distribution, epidemiology and genetic characterization of B. bigemina, B. bovis, and B. ovata in cattle in China as well as the prevalence of these protozoan agents were assessed.

Methods: A total of 646 blood specimens from cattle, dairy cattle and yaks from 14 provinces were collected and tested for the presence of the three Babesia species via a specific nested PCR assay based on the rap-1 and ama-1 genes. The PCR results were confirmed by DNA sequencing. Gene sequences and the genetic characterization were determined for selected positive samples from each sampling area.

Results: Of a total of 646 samples, 134 (20.7\%), 60 (9.3\%) and 10 (1.5\%) were positive for B. bovis, B. bigemina and $B$. ovata infections, respectively. Mixed infections were found in 7 of 14 provinces; 43 (6.7\%) samples were infected with B. bovis and B. bigemina. Three samples (0.5\%) exhibited a co-infection with B. bovis and B. ovata, and $6(0.9 \%)$ were infected with all three parasites. The rap-1a gene of $B$. bovis indicated a high degree of sequence heterogeneity compared with other published rap-1a sequences worldwide and was 85-100\% identical to B. bovis rap-1 a sequences in Chinese isolates. B. bigemina rap-1C and B. ovata ama-1 genes were nearly identical, with 97.8-99.3\% and 97.8-99.6\% sequence identity, respectively, in GenBank.

Conclusions: Positive rates of B. bovis and B. bigemina infection are somewhat high in China. The B. bovis infection in yaks was first reported. The significant sequence heterogeneity in different variants of the rap-1a gene from Chinese $B$. bovis isolates might be a great threat to the cattle industry if RAP-1a protein is used as immunological antigen against Babesia infections in China. The data obtained in this study can be used to plan effective control strategies against babesiosis in China.
\end{abstract}

Keywords: rap-1, ama-1, Molecular survey, Genetic diversity, Babesia

\footnotetext{
*Correspondence: luojianxun@caas.cn; yinhong@caas.cn

'State Key Laboratory of Veterinary Etiological Biology, Key Laboratory of Veterinary Parasitology of Gansu Province, Lanzhou Veterinary Research Institute, Chinese Academy of Agricultural Science, Xujiaping 1, Lanzhou, Gansu 730046, PR China

Full list of author information is available at the end of the article
} 


\section{Background}

Bovine babesiosis is a haemoparasitic disease caused by the intra-erythrocytic multiplication of apicomplexan protozoa, mainly Babesia bovis and B. bigemina, and is responsible for substantial mortality and morbidity rates and large economic losses in the livestock industry in tropical and subtropical regions. In China, the currently reported causative agents of bovine babesiosis are $B$. bigemina, B. bovis, B. ovata, B. major, B. orientalis and the recently described Babesia $U$ sp. Kashi, which was derived from Hyalomma anatolicum collected from the field [1-5]. Among these species, B. bovis and B. bigemina are the most common species affecting bovines and are usually present in co-infections in field animals. $B$. bigemina infection is normally characterized by a low level of parasitaemia, in contrast with $B$. bovis infection, which results in high parasitaemia and a more severe disease than $B$. bigemina. Both species are distributed worldwide and transmitted by the genus Rhipicephalus (Boophilus) microplus, which is one of the most widely distributed and economically important ticks. These two species of Babesia are considered to have the greatest economic impact on cattle health. B. major and B. ovata are transmitted by Haemaphysalis punctata and $H$. longicornis, respectively, whereas $B$. orientalis is transmitted by Rhipicephalus and has thus far been determined to be highly pathogenic only to buffalo [6]. B. divergens has been detected in anaemic human patients but has not been isolated in cattle in China [7].

The prevalence of babesiosis correlates with the geographic distribution and activity of vector ticks. Furthermore, changing environmental conditions, especially global warming, favours tick survival and reproduction and hence a significant increase in the abundance of ticks [8].

Traditionally, thin or thick smears stained with Giemsa can detect the presence of Babesia, based on the morphological examination. Useful to confirm acute cases, this method has limited value for chronic cases, where only low numbers of Babesia exist. Moreover, the species differentiation remains challenging (refer to $B$. bigemina co-existence with $B$. ovata, with the similar morphologies between them). Serological diagnostic tests such as IFAT and ELISA have been established for a number of Babesia species, with the drawback of serological cross-reactions between closely related species [9]. In contrast to these methods, the application of PCR-based molecular diagnostic tools allows direct, specific and sensitive detection of parasites as well as rapid, simultaneous detection and differentiation of co-infecting Babesia parasites similar morphologies and with same transmitted vector ticks in a given animal [10].

The clinical signs of babesiosis are usually characterized by fever, depression, haemolytic anaemia, haemoglobinuria, and icterus, and the disease can result in death in severe cases. The presence of these signs is due to the invasion and repeated rounds of asexual multiplication of the parasites in host erythrocytes. During the invasion process, several molecules, including Associatedmembrane-antigen-1 (AMA-1), Rhoptry-associated-protein-1 (RAP-1), Thrombospondin-related anonymous protein (TRAP) and spherical body proteins (SBPs), are secreted by the apical organelles and represent potential vaccine targets [11]. The highly conserved nature of the genes encoding these molecules suggests a potentially immunogenic function of these proteins.

AMA-1 protein is conserved among apicomplexan parasites [12] and has been characterized in the cattle Babesia species B. bovis [13], B. bigemina [14], B. ovata [15] and $B$. divergens [16].

In the genus Babesia, RAP-1 was initially described in $B$. bigemina [17] and was then characterized in all examined Babesia species, including B. bovis [18] and B. divergens [19]. The rap-1 gene family in all Babesia species studied is characterized by the presence of multiple copies of the gene arranged in tandem head to tail. Rap-1a has been identified in all Babesia species and is an informative marker for a broader phylogenetic analysis in the genus Babesia. Strict sequence identity of the rap-1b gene copies and a single polymorphic gene copy of rap-1c were only found in $B$. bigemina and $B$. motasi-like group-infected sheep. Rap$1 c$ is considered eligible for the characterization of field isolates of $B$. bigemina from different geographic areas $[20,21]$.

Rap-1 and ama-1 have been used as markers for the molecular diagnosis of cattle babesiosis [22-25]. The investigation of polymorphism in these genes and a comparative analysis of sequences between Babesia species infecting the same host in different geographic isolates may help to develop specific and sensitive molecular diagnostic tests for Babesia species infecting cattle around the world, and it is essential to develop effective preventive strategies before a disease outbreak. The prevalence of bovine babesiosis and the genetic diversity of these pathogens have not been well studied in China. The purpose of this study was to investigate the distribution of B. bigemina, B. bovis, and B. ovata in China and to determine the prevalence of these pathogens in cattle as well as to evaluate the genetic diversity of rap-1 (B. bovis, B. bigemina) and ama-1 (B. ovata), which encode potentially immunogenic proteins, among different isolates of these parasites in the world. Specific nested PCR assays were used to this end.

\section{Methods \\ Parasites}

$B$. bovis and B. bigemina were isolated from splenectomized cattle experimentally infected with Rhipicephalus (Boophilus) microplus ticks collected from Shanxian in 
Henan Province and Kunming in Yunnan Province, respectively, whereas B. ovata was isolated from Haemaphysalis longicornis ticks collected from pastures in Henan Province in China [4] and cryopreserved in liquid nitrogen at the Vector and Vector-borne disease (VVBD) laboratory of Lanzhou Veterinary Research Institute (LVRI), CAAS Lanzhou, China. The parasites were isolated by inoculating infected blood (10\% parasitaemia) cryopreserved in liquid nitrogen into haemoprotozoa-free splenectomized cattle. When parasitaemia was greater than $10 \%$, infected venous blood was collected into heparinized tubes, and the merozoites were purified for genomic DNA extraction and then used as a positive control.

\section{Blood sample collection}

A total of 646 field blood samples were randomly collected from clinically healthy cattle, dairy cattle or yak from 24 different locations in 14 Chinese provinces from June 2007 to July 2014 (Fig. 1): Inner Mongolia $(n=94)$, Gansu ( $n=94)$, Qinghai $(n=47)$, Xinjiang Uygur Autonomous Region $(n=50)$, Shaanxi $(n=21)$, Jilin $(n=23)$, Henan $(n=51)$, Chongqing $(n=53)$, Guangdong $(n=29)$, Guangxi $(n=32)$, Fujian $(n=25)$, Hebei $(n=33)$, Hainan $(n=37)$ and Yunnan $(n=57)$. All blood samples were collected in EDTA-coated Vacutainer tubes, transported to the laboratory with an ice pack and stored at $-20^{\circ} \mathrm{C}$.

\section{Genomic DNA sample preparation}

Genomic DNA was extracted from $300 \mu$ of each anticoagulated blood sample and handled using a QIAamp DNA Blood Kit (QIAGEN, Maryland, USA) following the manufacturer's instructions. Extracted DNA was eluted in $50 \mu \mathrm{l}$ of the elution buffer and then stored at $-20^{\circ} \mathrm{C}$ until use. Negative control DNA was isolated from the venous blood of uninfected cattle.

\section{Ethical approval}

This study was approved by the Animal Ethics Committee of the Lanzhou Veterinary Research Institute, Chinese Academy of Agricultural Sciences. All animals were handled in accordance with the Animal Ethics Procedures and Guidelines of the People's Republic of China.

\section{Primer design}

To obtain B. bovis, B. bigemina and B. ovata rap-1 and ama-1 gene sequences, the primer pairs of PCR and nested PCR for particular genes were designed based on sequences extracted from GenBank (under the following accession numbers: B. bovis rap-1a, AF030055-AF 030 062, FJ588009- FJ588013; B. bigemina rap-1c, AY146983AY146987, AF026272; B. ovata ama-1, AB634843) according to the multiple sequence alignments of each gene from Babesia species that infect ruminants (Table 1) for

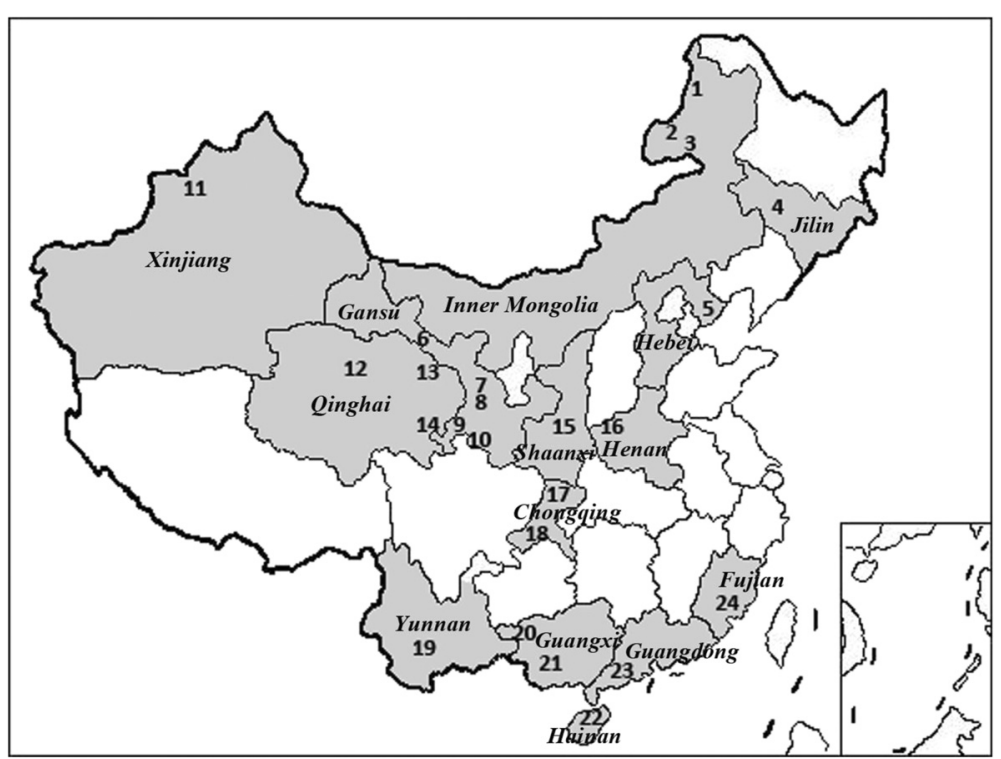

Fig. 1 Geographical distribution of the sampling sites in China.1. Xin Barag Left Banner (cattle, $n=53)$, 2. Manzhouli City (cattle, $n=27), 3$. Eergu'Na City (cattle, $n=14$ ), 4. Changchun City (cattle, $n=23), 5$. Tangshan City (dairy cattle, $n=33$ ), 6. Lintao Country (cattle, $n=14$ ), 7. Zhangye City (cattle, $n=14)$, 8. Zhuoni County of Gannan Tibetan Autonomous Prefecture (cattle, $n=20)$, 9. Lintan Country, Gannan Tibetan Autonomous Prefecture (yak, $n=24)$, 10. Luqu Country, Gannan Tibetan Autonomous Prefecture (yak, $n=22), 11$. Yili area (cattle, $n=50), 12$. Menyuan Country of Hui Autonomous County (yak, $n=14)$, 13. Haixing Country (cattle, $n=20), 14$. Gangcha Country (cattle, $n=13), 15$. Weiyuan Country (cattle, $n=21), 16$. Shanxian Country (cattle, $n=51)$, 17. Jiangjin area (cattle, $n=29)$, 18. Wanzhou area (cattle, $n=24), 19$. Puer City (cattle, $n=57), 20$. Chongzuo City (cattle, $n=12$ ), 21. Baise City (cattle, $n=20), 22$. Haikou City (dairy cattle, $n=37$ ), 23. Maoyuan City (dairy cattle, $n=29), 24$. Nanping City (cattle, $n=25)$ 
Table 1 Target gene, primer combinations, amplified conditions used for PCR amplifications

\begin{tabular}{|c|c|c|c|c|c|c|}
\hline Species & Target gene & PCR assay & Primer name & Sequence $\left(5^{\prime}-3^{\prime}\right)$ & $\operatorname{Tm}\left({ }^{\circ} \mathrm{C}\right)$ & Product size (bp) \\
\hline \multirow[t]{8}{*}{ B. bovis } & \multirow[t]{8}{*}{ rap-1a } & \multirow[t]{2}{*}{$P C R^{a}$} & \multirow[t]{2}{*}{ B.borap-1aF1/ B.borap-1aR1 } & ACGCGAATGGTTGCGTTTCAGA & \multirow[t]{2}{*}{55} & \multirow[t]{2}{*}{570} \\
\hline & & & & GGCTCAGCAACATTGGCTTTCAG & & \\
\hline & & \multirow[t]{2}{*}{$n P C R^{a}$} & \multirow[t]{2}{*}{ B.borap-1aF2/ B.borap-1aR2 } & GGTGATTACCACTACTTCGTCAC & \multirow[t]{2}{*}{55} & \multirow[t]{2}{*}{394} \\
\hline & & & & CGTACGAGGTCAAGCTACCGAGCAG & & \\
\hline & & \multirow[t]{2}{*}{$P C R^{b}$} & \multirow[t]{2}{*}{ B.borap-1afull-F/B.borap-1afull-R } & ATGAGAATCATTAGCGGCGTTGTCGGTT & \multirow[t]{2}{*}{64} & \multirow[t]{2}{*}{1698} \\
\hline & & & & TCAGAGGTATCCGGCGGTGTCTTCACCG & & \\
\hline & & \multirow[t]{2}{*}{$n P C R^{b}$} & \multirow[t]{2}{*}{ B.borap-1aseq-F/B.borap-1aseq-R } & TGTACGGATGCTTTACGATTGAC & \multirow[t]{2}{*}{59} & \multirow[t]{2}{*}{1358} \\
\hline & & & & AGTTGAGTCGTTAGACTGAGTGGTA & & \\
\hline \multirow[t]{5}{*}{ B. bigemina } & \multirow[t]{5}{*}{ rap-1c } & \multirow[t]{2}{*}{$P C R^{a}$} & \multirow[t]{2}{*}{ B.birap-1cF1/B.birap-1 cR ${ }^{\mathbf{c}}$} & AAGCAGCAGCCGTGGTACAAGCGTTGG & \multirow[t]{2}{*}{58} & \multirow[t]{2}{*}{657} \\
\hline & & & & TTACGACGATCGTTTGAAGTACTTC & & \\
\hline & & $n P C R^{a}$ & B.birap-1CF2/B.birap-1 cR ${ }^{c}$ & TGGCGAACTCGCAGACCAAGTAG & 60 & 274 \\
\hline & & $P C R^{b}$ & B.birap-1cF/B.birap-1 CR $^{\mathbf{c}}$ & ATGATTCACTACGCTTGCCTCA & 57 & 1530 \\
\hline & & $n P C R^{b}$ & B.birap-1cseq-F/B.birap-1 cR ${ }^{\mathbf{c}}$ & TTACGCTGCTTACTACAGCTTCA & 57 & 1054 \\
\hline \multirow[t]{6}{*}{ B. ovata } & \multirow[t]{6}{*}{$a m a-1$} & \multirow[t]{2}{*}{$P C R^{a}$} & \multirow[t]{2}{*}{ B.ovama-1 F1/B.ovama-1R1 c } & GGCAGGTGCCTGCGTGGCGATCG & \multirow[t]{2}{*}{62} & \multirow[t]{2}{*}{600} \\
\hline & & & & GAGCAACAACAGCGCCGTAGTAATCACG & & \\
\hline & & \multirow[t]{2}{*}{$n P C R^{a}$} & \multirow[t]{2}{*}{ B.ovama-1 F2/ B.ovama-1R2 } & TGATATCGATATCGACCTTGATTC & \multirow[t]{2}{*}{62} & \multirow[t]{2}{*}{310} \\
\hline & & & & GAGCTGTCACCATTGTCCTTAACAC & & \\
\hline & & $P C R^{b}$ & B.ovama-1seqF/B.ovama-1R1 ${ }^{\mathbf{c}}$ & GATACGAGGCTGTCGGTAGC & 62 & 1234 \\
\hline & & $\mathrm{nPCR} \mathrm{R}^{\mathrm{b}}$ & B.ovama-1seqF/B.ovama-1R2 & & 58 & 1041 \\
\hline
\end{tabular}

${ }^{\mathrm{a}}$ For screening analysis

${ }^{\mathrm{b}}$ For sequencing analysis

cbold data: The sequences of these primeres appeared twice or more times

screening and sequencing. The primers targeted the rap$1 a$ (B. bovis), rap-1c (B. bigemina), and ama-1 (B. ovata) genes for amplification of the full length of or part of the homologous gene of interest. The specificity of each primer was evaluated against the DNA of various Babesia species.

\section{Detection of field samples with two sets PCR assays}

The specificity of both primer sets was initially tested to amplify the targeted gene of interest, and other parasitic pathogens were used as controls. All of the DNA field samples were then amplified to screen for the presence of parasites. The two sets of primers were used to amplify the shorter fragment of each gene (Table 1). The PCR amplification was conducted in a final volume of $25 \mu \mathrm{l}$, composed of $12.5 \mu \mathrm{l}$ of Premix $\mathrm{Taq}^{\mathrm{Tx}}$ (TaKaRa $\mathrm{Taq}^{\mathrm{ma}}$ Version 2.0 plus dye), $0.5 \mu \mathrm{l}(20 \mathrm{pmol})$ of each external primer, $3 \mu \mathrm{l}$ of the template DNA sample, $8.5 \mu \mathrm{l}$ of double-distilled water and $1 \mu \mathrm{l}$ of positive control DNA. The amplification parameters for PCR were an initial denaturation at $94{ }^{\circ} \mathrm{C}$ for $5 \mathrm{~min}$ followed by $35 \mathrm{cy}-$ cles of denaturation at $94{ }^{\circ} \mathrm{C}$ for $30 \mathrm{~s}$, annealing at $55{ }^{\circ} \mathrm{C}$ or $58{ }^{\circ} \mathrm{C}$ or $62{ }^{\circ} \mathrm{C}$ for $45 \mathrm{~s}$, and extension at $72{ }^{\circ} \mathrm{C}$ for $1 \mathrm{~min}$, with a final extension at $72{ }^{\circ} \mathrm{C}$ for $10 \mathrm{~min}$. Each product from the first PCR reaction $(1 \mu \mathrm{l})$ was then added to the second PCR mixture, and Ex Taq Hot-Start
DNA polymerase (TaKaRa) was used to amplify the internal fragment using the internal primers.

The expected size of the amplicons was analysed on $1.5 \%$ agarose gels with GoldView I nucleotide stains (Solarbio) and visualized under UV illumination.

For the sequencing of obtained positive samples for the study of the phylogenetic analysis among different isolates, both sets of primers were used to amplify full-length or longer sequences of each gene from randomly selected positive samples. PCR mixtures and amplification were performed as described above using the appropriate annealing temperatures. The amplicons (approximately $1.4 \mathrm{~kb}$ for $B$. bovis rap-1a and approximately $1 \mathrm{~kb}$ for $B$. bigemina rap-1c and $B$. ovata ama-1) from secondary PCR were used for phylogenetic analysis.

\section{Cloning and sequencing}

The PCR products from selected field-positive samples (11 samples for B. bovis rap-1a, 10 samples for B. bigemina rap-1c and 3 samples for $B$. ovata ama-1) were purified using a MiniBEST DNA Fragment Purification Kit (TaKaRa) and cloned into pGEM ${ }^{\circ}-\mathrm{T}$ Easy Vector Systems (Promega) and then transformed into Trans $5 \alpha$ Chemically Competent Cells (TransGen Biotech) according to the manufacturers' instructions. Colonies were selected by direct colony PCR using vector primers. 
Multiple sub-colonies from each sample were selected, cultured in $3 \mathrm{ml} \mathrm{LB}$ broth and sequenced. The long inserts were sequenced by successively designing internal primers.

\section{Bioinformatics analysis}

All sequences obtained in this study were subjected to a blast search on the NCBI website (http://blast.ncbi.nlm.nih.gov/Blast.cgi) using the BLASTn program.

Multiple sequence alignments were analysed using the ClustalW 2.0.12 software ClustalW: Multiple alignment. Phylogenetic analysis was performed using the MEGA 6.06 software [26]. The three different genes were analysed using the "neighbour-joining" method based on genetic distances. Phylogenetic trees were constructed from other isolates of $B$. bovis, B. bigemina and B. ovata by including selected gene sequences and related database sequences in GenBank to show relationships between different isolates from our study and other regions in the world.

\section{Statistical analysis}

The $95 \%$ confidence intervals (95\% CIs) for the overall prevalence values of each Babesia species were calculated using IBM SPSS Statistics version 19.0.

\section{Results}

PCR detection of three Babesia species from field samples All primers amplified only their respective target sequence for individual Babesia species; no cross reaction was observed. No amplification was observed when cattle genomic DNA and water were used as controls (data not shown). Field blood samples extracted from a total of 646 cattle, dairy cattle or yak from 14 Chinese provinces were detected with both nPCR assays. The first round of nested PCR assays screening primers specific for the rap-1 or ama-1 genes for the presence of the three Babesia species of interest were developed to detect $B$. bovis, $B$. bigemina and $B$. ovata in cattle samples. The samples were amplified with a length of $\sim 394$ bp for B. bovis, $\sim 277 \mathrm{bp}$ for $B$. bigemina and $\sim 310 \mathrm{bp}$ for $B$. ovata for the genes of interest, respectively. The results of the nested PCR amplification for positive sample screenings are summarized in Table 2. In summary, cattle blood samples infected with $B$. bovis were found in 12 of 14 surveyed provinces, and 8 provinces of 14 exhibited B. bigemina infections. Only cattle from Henan Province showed B. ovata infection. Babesia DNA was found in 134 (B. bovis), 60 (B. bigemina), and 10 (B. ovata) of the 646 cattle, dairy cattle or yak from all regions. The total infection rates indicated by nPCR were $20.7 \%$ for B. bovis, $9.3 \%$ for B. bigemina and $1.5 \%$ for $B$. ovata. The total $B$. bovis infection rate in yaks was $13 \%(8 / 60)$, with $27 \%(6 / 22)$ in Luqu Country and $8.3 \%$ (2/24) in Lintan Country in Gansu province, respectively. No positive sample was found in yaks collected from Qinghai province. Of all these positive samples, $43 \mathrm{had}$ double infections of $B$. bovis and B. bigemina in seven provinces; three and six samples had $B$. bovis $+B$. ovata double infections and B. bovis $+B$. bigemina $+B$. ovata infections, respectively; and triple infections in which all

Table 2 Detection results of B. bovis, B. bigemina and B. ovata in field blood samples

\begin{tabular}{|c|c|c|c|c|c|c|c|c|c|}
\hline \multirow[t]{2}{*}{ Province } & \multirow{2}{*}{$\begin{array}{l}\text { Date } \\
\text { Year/month }\end{array}$} & \multirow{2}{*}{$\begin{array}{l}\text { No. of } \\
\text { samples }\end{array}$} & \multicolumn{3}{|c|}{ Positive rate (\%) } & \multicolumn{4}{|c|}{ Mix Positive rate (\%) } \\
\hline & & & B. bovis & B. bigemina & B. ovata & B. bo+B.bi & B. $b i+$ B. ov & B. $b o+$ B. ov & B. bo + B. bi+B.ov \\
\hline Inner Mongolia & 2013.6 & 94 & $1(1)$ & $1(1)$ & 0 & 0 & 0 & 0 & 0 \\
\hline Gansu & 2013.5 & 94 & $22(23.4)$ & 0 & 0 & 0 & 0 & 0 & 0 \\
\hline Qinghai & 2013.1 & 47 & $3(6.4)$ & 0 & 0 & 0 & 0 & 0 & 0 \\
\hline Xinjiang & 2014.7 & 50 & 0 & 0 & 0 & 0 & 0 & 0 & 0 \\
\hline Shaanxi & 2013.7 & 21 & 0 & 0 & 0 & 0 & 0 & 0 & 0 \\
\hline Jilin & 2008.6 & 23 & $1(4.3)$ & 0 & 0 & 0 & 0 & 0 & 0 \\
\hline Henan & 2007.5 & 51 & $28(54.9)$ & $13(25.5)$ & $10(19.6)$ & $11(21.6)$ & 0 & $3(5.9)$ & $6(11.8)$ \\
\hline Chongqing & 2011.5 & 53 & $13(24.5)$ & $3(5.7)$ & 0 & $2(3.8)$ & 0 & 0 & 0 \\
\hline Guangdong & 2011.6 & 29 & $1(3.4)$ & 0 & 0 & 0 & 0 & 0 & 0 \\
\hline Guangxi & 2011.4 & 32 & $12(37.5)$ & $2(6.3)$ & 0 & $2(6.3)$ & 0 & 0 & 0 \\
\hline Fujian & 2012.5 & 25 & $15(60)$ & $6(24)$ & 0 & $5(20)$ & 0 & 0 & 0 \\
\hline Hebei & 2008.4 & 33 & $1(3)$ & $1(3)$ & 0 & (1 (3) & 0 & 0 & 0 \\
\hline Hainan & 2013.5 & 37 & $16(43.2)$ & $23(62.2)$ & 0 & $15(40.5)$ & 0 & 0 & 0 \\
\hline Yunnan & 2014.7 & 57 & $21(36.8)$ & $11(19.3)$ & 0 & $7(12.3)$ & 0 & 0 & 0 \\
\hline Total & & 646 & $134(20.7)$ & $60(9.3)$ & $10(1.5)$ & $43(6.7)$ & 0 & $3(0.5)$ & $6(0.9)$ \\
\hline
\end{tabular}

B. bo: B. bovis

B. bi: B. bigemina

B. ov: B. ovata 
Babesia species were detected occurred only in samples from Henan Province. The highest prevalence of B. bovis and B. bigemina infections occurred in Fujian and Hainan Provinces, respectively.

Sequence variation of rap-1 and ama-1 genes in the Chinese B. bovis, B. bigemina and B. ovata isolates

The amplification of the rap-1 and ama-1 genes using the appropriate primers (B.borap-1aseq-F/R; B.birap1cseq-F/B.birap-1cR and B.ovama-1seqF/B.ovama-1R2, Table 1) produced three different amplicons of 1358 , 1057 and $1041 \mathrm{bp}$, respectively. The size of the amplicons might correspond to the expected length of rap-1 or ama-1. Ten clones per sample containing inserts were sequenced for $B$. bovis, $B$. bigemina and $B$. ovata, all of them exhibiting homologous rap-1 or ama-1 genes in the databases. A representative sequence from each sample was selected based on the sequence alignments of ten clones. Sequencing and sequence analysis confirmed that the nPCR assays were specific for the target genes and Babesia species.

\section{B. bovis rap-1a}

Sequence alignment of rap-1a genes from 11 Chinese strains demonstrated 99 nucleotide substitutions and 36 amino acid modifications (non-synonymous) that were affected by 42 of these nucleotide substitutions. Among these substitutions, 58 nucleotide substitutions were found in Henan strains 1 and 3 (Additional file 1a). The common molecular features of the RAP-1 protein in all studied Babesia spp. indicated the presence of patches of conserved motifs in the first $\mathrm{N}$-terminal of approximately 300 amino acids. In this study, the amplified region (463 aa) began at aa 103 and included an N-terminal conserved region from aa 1 to aa 211 compared with the full-length RAP-1a sequence of B. bovis of 565 aa). Seven modifications between aa 1 and aa 211 (N-terminal) and 10 modifications between aa 396 and aa 463 were identified. The differences (20 aa modifications) in the protein sequences were mostly limited to a specific region composed of repeated sequences (23 aa per repeat) between aa 212 and aa 395 (Additional file 1b). In the rap-1a genes, these regions were localized at the $\mathrm{C}$-terminal of the gene, starting between 634 and 1185 bp in the sequences amplified in this study, with a total length of 552 bp (Additional file 1a).

Thirteen single substitutions at the first and 13 at the second positions were synonymous (26/36), but only four substitutions at the third position were synonymous (4/36), whereas double substitutions at the first and third or second and third positions were synonymous (6/36) (Additional file 1b).

\section{B. bigemina rap-1C}

Precise sequences of the rap-1c derived from 10 strains demonstrated 16 substitutions at different positions (Additional file 2). Six amino acid modifications affected seven codons (non-synonymous) by nucleotide substitutions among the isolates. Four amino acid modifications (nucleotide positions: 602, 608, $749+751$ and 1024) occurred in two Yunnan isolates. Two substitutions (602, 608 ) at the first position, two substitutions at the first and third positions $(749+751)$ and one substitution (1024) at the third position, as well as one aa change (nucleotide position: 889, substitution at the second position), occurred in the Chongqing isolate. Another aa modification (nucleotide position: 969, substitution at the third position) occurred in the HAN2 isolate (Additional file 2). Nine substitutions were synonymous.

The per cent identity among the Chinese isolates of $B$. bovis and B. bigemina based on the rap-1a and rap-1c genes was determined and is shown in Table 3.

\section{B. ovata ama-1}

The sequences of the $a m a-1$ gene from three isolates of the Henan strains were obtained and revealed $100 \%$ identity between isolates 1 and 2, whereas 12 nucleotide substitutions involving a 1 aa modification were found in isolate 3 compared with the other two isolates.

\section{Phylogenetic analysis}

Three phylogenetic trees were constructed based on $B$. bovis rap-1a $(n=11), B$. bigemina rap-1c $(n=10)$ and $B$. ovata ama-1 $(n=3)$ gene sequences determined in the present study by the neighbour-joining method using the software MEGA6.06. These gene sequences were deposited in GenBank in various countries.

\section{B. bovis rap-1a}

The rap-1 phylogenetic tree indicated that the rap-1a sequences from our study formed five clades that were generally distinct from the rap-1a sequences from other countries, with several sub-branches (Fig. 2). Two Yunnan isolates formed a sub-branch in the first clade, forming a sister clade with the B. bovis rap-1a sequences from the USA, Argentina, Brazil and Uruguay. The isolates from Fujian, Gansu, and Guangxi Provinces formed the second clade, clustered with several sub-branches. The Guangxi isolate was more closely related to $B$. bovis isolate KZN-B13, which is from South Africa, whereas isolates from Chongqing and Hainan were also in this clade but formed separate, single branches. The rap-1a sequence (Henan-2) from Henan cattle formed a single clade (clade 3), whereas two other Henan isolates formed a different single clade (clade 4). The previously published rap-1a sequences of $B$. bovis of cattle from Israel formed a separate clade (clade 5). In general, the 
Table 3 Percent identity of B. bovis rap-1a and B. bigemina rap-1C gene sequences of Chinese isolates deduced after CLUSTAL W alignment

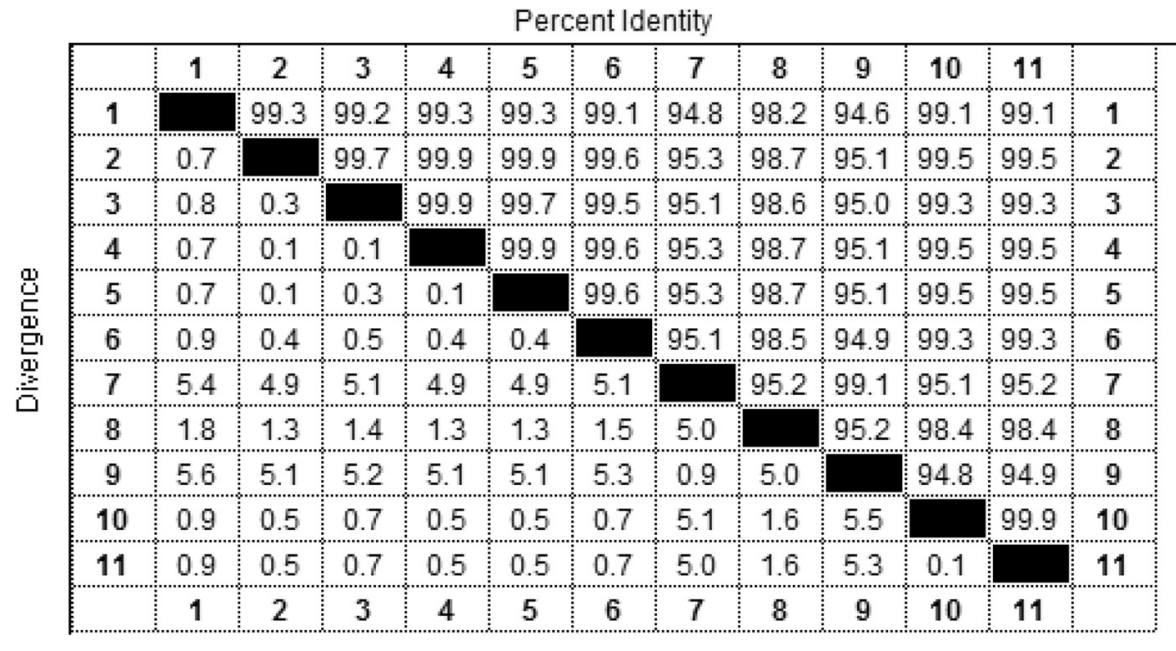

\author{
B.bovis Chongqing KT 318580 \\ B.bovis Fujian-1 KT312806 \\ B.bovis Fujian-2 KT312807 \\ B.bovis Gansu KT318579 \\ B.bovis Guangxi KT 312808 \\ B.bovis Hainan KT312809 \\ B.bovis Henan-1 KT312810 \\ B.bovis Henan-2 KT312811 \\ B.bovis Henan-3 KT312812 \\ B.bovis Yunnan-1 KT312813 \\ B.bovis Yunnan-2 KT312814
}

\begin{tabular}{|c|c|c|c|c|c|c|c|c|c|c|c|}
\hline \multicolumn{12}{|c|}{ Percent Identity } \\
\hline & 1 & 2 & 3 & 4 & 5 & 6 & 7 & 8 & 9 & 10 & \\
\hline 1 & & 99.7 & 99.8 & 99.9 & 99.8 & 99.9 & 99.6 & 99.9 & 99.1 & 99.1 & 1 \\
\hline 2 & 0.3 & & 99.7 & 99.8 & 99.7 & 99.8 & 99.5 & 99.8 & 99.1 & 99.1 & 2 \\
\hline 3 & 0.2 & 0.3 & & 99.9 & 99.8 & 99.9 & 99.6 & 99.9 & 99.1 & 99.1 & 3 \\
\hline 4 & 0.1 & 0.2 & 0.1 & & 99.9 & 100.0 & 99.7 & 100.0 & 99.2 & 99.2 & 4 \\
\hline 5 & 0.2 & 0.3 & 0.2 & 0.1 & & 99.9 & 99.6 & 99.9 & 99.1 & 99.1 & 5 \\
\hline 6 & 0.1 & 0.2 & 0.1 & 0.0 & 0.1 & & 99.7 & 100.0 & 99.2 & 99.2 & 6 \\
\hline 7 & 0.4 & 0.5 & 0.4 & 0.3 & 0.4 & 0.3 & & 99.7 & 99.0 & 99.0 & 7 \\
\hline 8 & 0.1 & 0.2 & 0.1 & 0.0 & 0.1 & 0.0 & 0.3 & & 99.2 & 99.2 & 8 \\
\hline 9 & 0.9 & 1.0 & 0.9 & 0.8 & 0.9 & 0.8 & 1.0 & 0.8 & & 100.0 & 9 \\
\hline 10 & 0.9 & 1.0 & 0.9 & 0.8 & 0.9 & 0.8 & 1.0 & 0.8 & 0.0 & & 10 \\
\hline & 1 & 2 & 3 & 4 & 5 & 6 & 7 & 8 & 9 & 10 & \\
\hline
\end{tabular}

B.bigemina Chongqing KT312796

B.bigemina Fujian-1 KT312799

B.bigemina Fujian-2 KT312800

B.bigemina Guangxi-1 KT312797

B.bigemina Guangxi-2 KT312798

B.bigemina Hainan-1 KT312802

B.bigemina Hainan-2 KT312803

B. bigemina Hebei KT312801

B.bigemina Yunnan-1 KT312804

B.bigemina Yunnan-2 KT312805 results show the presence of high heterogeneity among the rap-1 sequences of different isolates of $B$. bovis species worldwide (Fig. 2).

\section{B. bigemina rap-1c}

Three distinct clades were present (Fig. 3) in the sequences of the B. bigemina rap-1c phylogenetic tree. All of the rap-1c sequences of $B$. bigemina from ten Chinese isolates were within the first clade with five sister branches: the first branch comprised Fujian, Guangxi and Hainan-1 rap-1c; the fifth comprised two Yunnan isolates; and the other three branches contained only one isolate. The previously published rap-1c sequences of $B$. bigemina from Argentinian and Brazilian cattle formed clade 2, whereas the rap-1c sequences from Spanish, Kenyan and Mexican cattle formed clade 3 (Fig. 3).

\section{B. ovata ama-1}

A limited number of $B$. ovata ama-1 sequences were found in GenBank. A phylogenetic tree was constructed based on all B. ovata ama-1 sequences deposited in GenBank, and three sequences obtained in this study were used (Fig. 4). All the ama-1 sequences from $B$. ovata formed two main clades. Two Henan isolates clustered in a sister branch with the B. ovata ama-1 from Japan, Mongolia and Thailand, forming the first main clade, and another $B$. ovata Henan-2 isolate formed a separate clade (Fig. 4).

\section{Nucleotide accession numbers}

All sequences, including 11 for $B$. bovis rap-1a, 10 for $B$. bigemina rap-1c and 3 for $B$. ovata ama- 1 genes, were deposited in GenBank with the following accession numbers: 9 (KT312806- KT312814) and 2 (KT318579- 


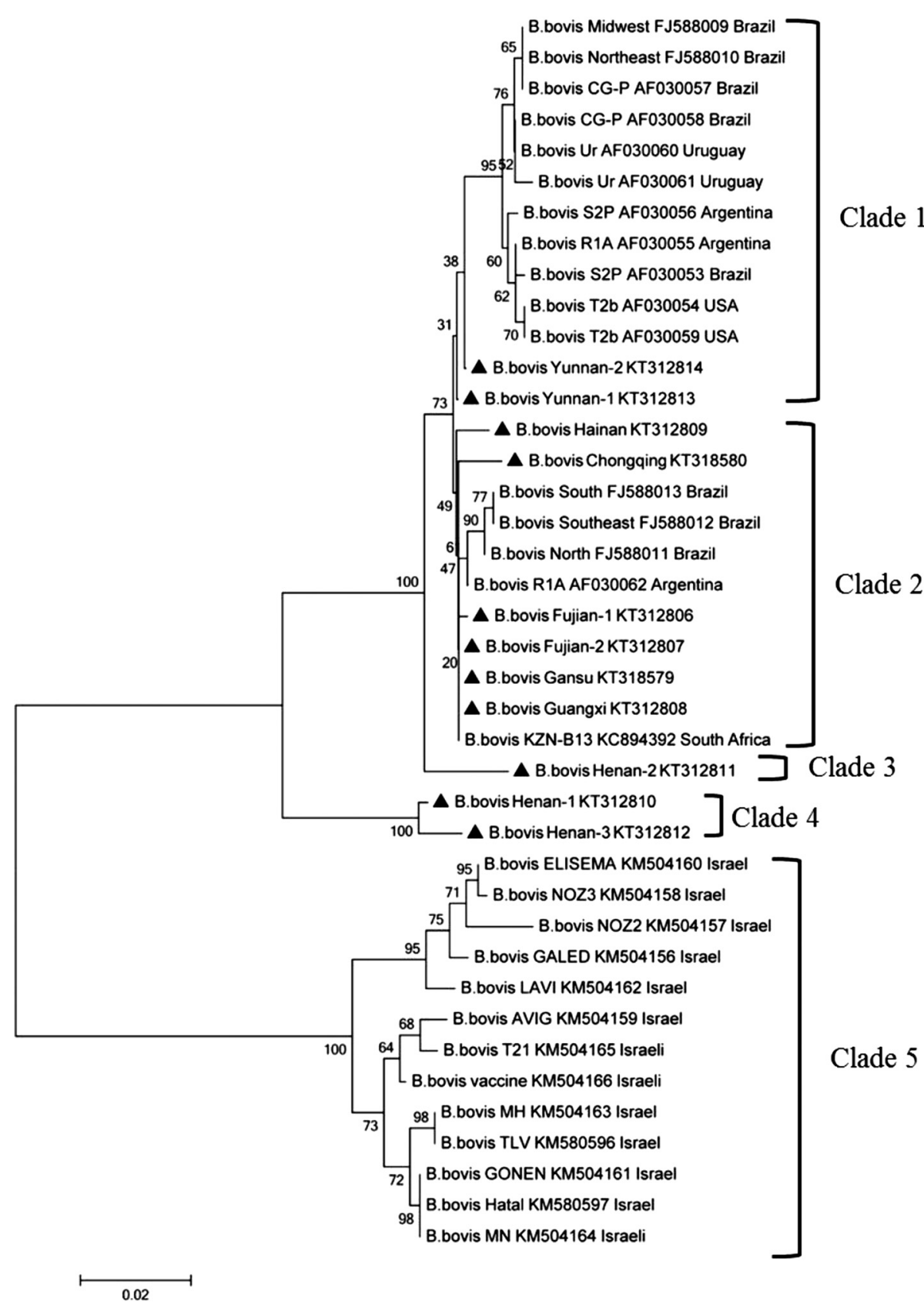

Fig. 2 Phylogenetic tree of the nucleotidic sequences of B. bovis rap-1a and all sequences of this gene deposited in GenBank from different countries, the accession numbers and countries are shown after isolate name. The rap-1a sequences obtained in this study were indicated with bold triangle. The tree was inferred using the neighbor joining method of MEGA6.06, bootstrap values are shown at each branch point. Numbers above the branch demonstrate bootstrap support from 1000 replications. All sites of the alignment containing insertions-deletions, missing data were eliminated from the analysis (option "complete deletion")

KT318580) sequences of $B$. bovis rap-1a from seven provinces (two from Fujian, one from Guangxi, one from Hainan, three from Henan, two from Yunnan, one from Gansu and one from Chongqing), 10 (KT312796KT312805) sequences of B. bigemina rap-1c from six provinces (one from Chongqing, two from Guangxi, two from Fujian, one from Hebei, two from Hainan, and two from Yunnan) and 3 (KT312793-KT312795) sequences of B. ovata ama-1 from only Henan Province.

\section{Discussion}

In China, babesiosis is caused by various protozoan parasites of the genus Babesia. Many of these agents are 


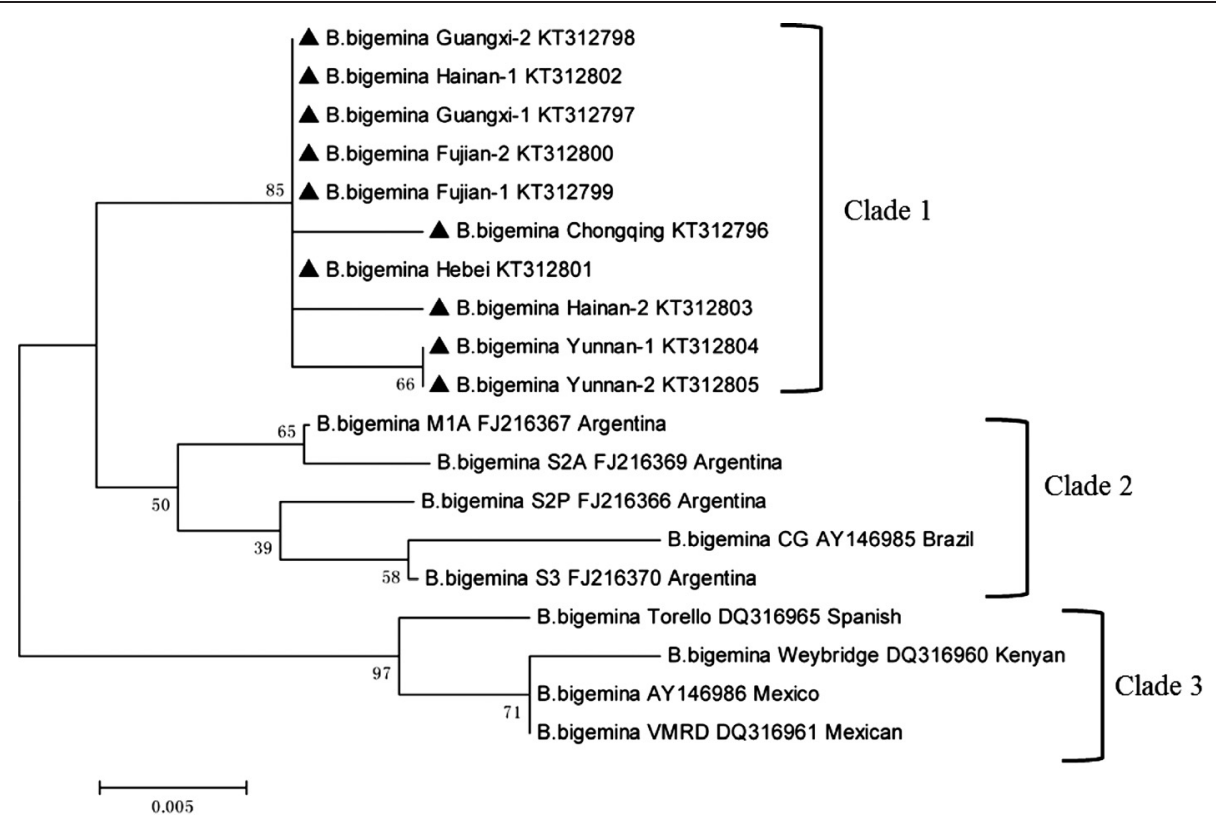

Fig. 3 Phylogenetic tree of the nucleotidic sequences of B. bigemina rap-1C and all sequences of this gene deposited in GenBank from different countries, the accession numbers and countries are shown after isolate name. The rap-1c sequences obtained in this study were indicated with bold triangle. The tree was inferred using the neighbor joining method of MEGA6.06, bootstrap values are shown at each branch point. Numbers above the branch demonstrate bootstrap support from 1000 replications. All sites of the alignment containing insertions-deletions, missing data were eliminated from the analysis (option "complete deletion")

highly pathogenic for ruminant cattle, buffalo, and yak $[2,27]$. Cattle grazing areas are distributed in a broad range in China. Ruminant babesiosis, which is mainly caused by the pathogenic agents $B$. bovis and $B$. bigemina, is considered one of the most prevalent tickborne diseases and has an important economic impact in China. Four B. ovata isolates (Lushi, Ningxian, Zhangjiachuan and Wenchuan) from Henan, Guansu and Sichuan Provinces have been reported in China [4]. However, the distribution of this pathogen may be much wider, depending on the distribution of its transmitted vector H. longicornis [28].

Previous studies reported the prevalence of cattle Babesia species in field blood collected from different Chinese sites, and the results showed that the distribution of the Babesia species varied considerably in the different areas. This variability may have been caused by the distribution of the different tick vectors from different sampling sites [29, 30].

In our study, the prevalence of three cattle babesial pathogens was investigated in a broad geographic range in the field. A total of 646 bovine blood samples were collected from 14 provinces located in northeast, northwest, central and southern China and were analysed using nested PCR assays. The findings revealed that $B$. bovis was the most widespread bovine haemoprotozoan species in Chinese cattle, but B. bigemina was less widespread, similar to the results of a previous study of these parasites. However, the positive rate of Babesia parasites was apparently higher than in previous findings using multiplex PCR and LAMP assays based on the gene target ITS $[27,28]$.

It is worth mentioning that few reports have described the B. bigemina infection in yaks from in Xinjiang Uygur Autonomous Region, northwestern China and Tianzhu Tibetan Autonomous County (TTAC), Gansu Province, northwest China and Nepal [31-33], however, the infection detection was only based on the serological survey, the identification at species level needs to be further confirmed. The recent report confirmed the B. bigemina infection in yaks in India using a sensitive PCR based on the small-subunit rRNA (SS rRNA) gene of B. bigemina and by further sequence analysis as well as by studying its restriction profile of the amplified fragment [34]. No positive samples about $B$. bigemina infection in yaks were found in our study. For the infection and prevalence of $B$. bovis in yaks, no scientific survey has been conducted so far. In the present study, the detected yaks with $B$. bovis positive were from Gannan Tibetan Autonomous Prefecture, Luqu and Lintan Countries, located at east and northeast edges of the Qinghai Tibet Plateau, Gansu Province of China, respectively. The weather is cold, damp and with low oxygen content, altitude of 2900-4200 m and 2200-3900 m, they have hypoxia tolerance and cold-resistant ability in the environment. Detection and molecular confirmation of B. bovis infection 


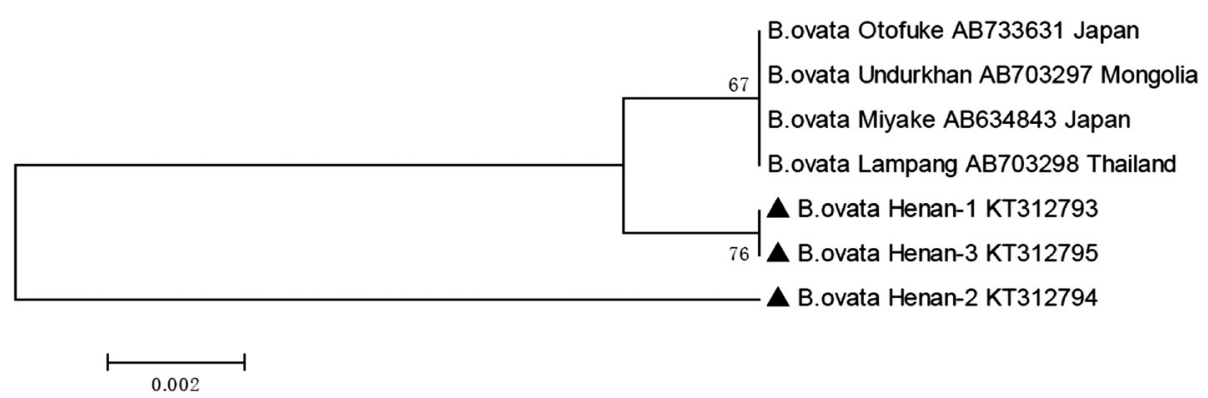

Fig. 4 Phylogenetic tree of the nucleotidic sequences of B. ovata ama-1 and all sequences of this gene deposited in GenBank from different countries, the accession numbers and countries are shown after isolate name. The ama-1 sequences obtained in this study were indicated with bold triangle. The tree was inferred using the neighbor joining method of MEGA6.06, bootstrap values are shown at each branch point. Numbers above the branch demonstrate bootstrap support from 1000 replications. All sites of the alignment containing insertions-deletions, missing data were eliminated from the analysis (option "complete deletion")

in yaks was first reported. The studies of importance for the tick vector, $R$. (B.) microplus transmitted Babesia to yaks is limited. Recent study indicated that the $R$. (B.) microplus could be collected from field at high altitude of 1800-2200 m [35]. Saravanan et al. [34] reported the yaks are moved to higher altitude of more than $4500 \mathrm{~m}$ during summer and brought back during winter. Due to animal migration, the $R$. (B.) microplus are likely to transfer to those regions with higher altitudes. However, the presence and survival of $R$. (B.) microplus at Luqu and Lintan Countries with higher altitudes is unknown. Therefore the potential appearance of babesiosis of $R$. (B.) microplus in yaks needs to be further studied. In addition, the sharing of common grazing lands with cattle in survey areas results in common vector born B. bovis infections in yaks might be another explanation.

The widespread co-existence (7 provinces) of B. bovis and B. bigemina implies the presence and distribution of the same tick vectors $(R .(B$.$) microplus) in the surveyed$ areas. $R$. (B.) microplus is one of the most important tick species causing babesiosis in cattle and is widespread in China. Approximately 24 provinces have reported the occurrence of this tick species [36].

B. ovata infection was found in only 10 samples from Henan Province, which is unusual because this parasite is reportedly widespread in China [4]. In addition, the tick vector of $B$. ovata is $H$. longicornis, which is also a prevalent tick species and is widely distributed in Jilin, Hebei, Gansu, and Shaanxi Provinces and in Chongqing city [36]. The lower infection rate of B. ovata in our study is thus most likely a result of sampling different regions, depending on the marker used for the molecular detection or the lower sensitivity of the PCR method used for Babesia species.

The overall infection rates of B. bovis, B. bigemina and B. ovata were $20.7 \%$ (95\% CI $=10.8-32.6), 9.3 \%$ (95\% $\mathrm{CI}=2.8-20.6)$ and $1.5 \%(95 \% \mathrm{CI}=0-4.2)$, respectively, in blood samples using the nested PCR assay (Table 2). These rates are comparable to other studies using multiplex PCR and LAMP assays; positive rates for B. bovis and $B$. bigemina were 7.7 and $5.8 \%$, respectively, with multiplex PCR [30] and 13.5 and $7.3 \%$, respectively, with LAMP [29]. Therefore, the wide prevalence of bovine blood samples positive for B. bovis and B. bigemina, as well as co-infections of two parasites in this study, was not surprising. The results suggest that the nested PCR assay developed based on the molecular markers rap-1 and ama-1 in our study can be considered as a potential diagnostic method for epidemiological investigations.

Functional protein secretion during the parasite's asexual growth cycle in the blood stage might be an effective control strategy for babesiosis [37]. The ama-1 and rap-1 gene products are considered potential antigen candidates because of their role in red blood cell invasion [38, 39]. Understanding the genetic characteristics and evolution of these genes is essential for designing a protective antigen against babesiosis.

In $B$. bovis, conservation of the gene sequence among geographically distinct isolates of rap-1a would be advantageous for the development of a diagnostic antigen in China, and immune control strategies against B. bovis in China could be designed in light of the genetic diversity of this parasite. In this study, we therefore investigated the sequence polymorphism of these genes from randomly selected positive samples from distinct geographical provinces and compared these with genes from other countries worldwide. Although rap-1 is a multiplegene family, only the rap-1a gene type was described in B. bovis [18]. The molecular features of the rap-1 gene family show that the N-terminal region of the RAP-1 sequence is more highly conserved among babesial parasites than the C-terminal region, and several sequences encode conserved or degenerate repeats located at the $\mathrm{C}$-terminal in many Babesia species, including B. bovis [40]. 
In our study, the amplification range for the $B$. bovis rap-1a gene was focused on the fragment starting from $305 \mathrm{bp}$ of the full-length (1358 bp) gene to the 3 ' end. The sequence analysis demonstrated sequence diversity, but some conserved features of the rap-1 family were also evident: a conserved 14 amino acid motif (PLTLPNPYQLDAAF) and several shorter conserved oligopeptide motifs (YKTYL) close to the $\mathrm{N}$-terminal region (Additional file $1 \mathrm{~b}$ ). Manual alignment revealed 8 degenerate repeats in a variable region of the C-terminal, consisting of 69 nt per repeat that corresponded to the published B. bovis RAP-1a sequence (GenBank accession number: ACM4406). Analysis of these repeat sequences in our study revealed 20 aa differences among 11 different isolates (Additional file 1b). The results suggest that targeting a common region to produce a protective antigen from the $\mathrm{C}$-terminal region of protein might be ineffective because of the great genetic variation in rap-1a among different isolates, even though these repeats were found to contain the predominant predicted linear protein B-epitope in the RAP-1a sequences. In general, these repeats are thought to mediate functions involved in parasite survival and immune evasion [41]. The phylogenetic analysis of rap-1a sequences from different worldwide isolates also indicated genetic variation; $11 \mathrm{~B}$. bovis Chinese isolates were segregated into three separate clades (Fig. 2). In contrast, a similar previous study showed the rap-1a sequences of distinct isolates from the same country were located on the same clade of the tree and reported a high degree of conservation of sequences at the nucleotide and amino acid levels among isolates $[22,24]$. The characterization of the genetic diversity of this region in $B$. bovis rap-1a Chinese isolates could be used as an informative marker for a broader phylogenetic analysis of the genus Babesia.

The ClustalW alignment of multiple rap-1a sequences of all $B$. bovis Chinese isolates shows that the identity among $B$. bovis isolates is greater than $99 \%$, except for the Henan isolates. The per cent identity of three Henan isolates compared to the other eight $B$. bovis isolates was 94.6-95.2\% (Table 3); the rap-1a sequences of Henan isolates showed a higher degree of heterogeneity. A comparison of the rap-1 sequences of Chinese isolates with published rap-1a sequences from South Africa, Argentina, USA, Brazil and Israel revealed identities ranging from $85-100 \%$ [22, 42-44].

From many perspectives, sequence analysis of the rap1 sequences obtained (1358 bp) revealed less sequence heterogeneity at the $5^{\prime}$ end of the rap-1 gene and greater sequence heterogeneity in the middle of the repeated region and at the 3' end of the rap-1 gene in Chinese isolates, as well as in isolates elsewhere in the world. These findings suggest a lack of conservation of the C-terminal repeat region and indicate that B-cell-rich epitopes could be used as a species-specific diagnostic ELISA antigen for the detection of antibodies to B. bovis.

The presence of only one rap-1c gene copy located at the $3^{\prime}$ end of the rap-1 locus was found only in the $B$. bigemina genome in cattle, and rap-1c has proven to be transcribed but not translated [39] and could thus be used as specific molecular marker [20]. In our study, the genetic polymorphism of the rap-1c gene from $B$. bigemina isolates from China and other countries was analysed. The sequences show limited polymorphism among different Chinese isolates, with a maximum of 6 amino acid substitutions (Additional file 2). The per cent identity of $10 \mathrm{~B}$. bigemina Chinese isolates was high, ranging from 99 to $100 \%$ (Guangxi isolate with Hainna-1and Hebei and two Yunnan isolates) (Table 3). The phylogenetic analysis indicated that the rap-1c sequences of the B. bigemina isolates deduced in the present study were clustered together in several branches. Furthermore, a comparison of the rap$1 c$ in Chinese B. bigemina field isolates with sequences derived from Brazilian and Mexican B. bigemina isolates revealed that the total sequence identity among the isolates ranged from 97.8 to $99.3 \%$ [39].

Our findings agree with previous studies that demonstrated that the nucleotide alignment of rap-1c from field isolates had an overall identity ranging from 99.0 to $100 \%$, with the largest number of changes in the amino acid sequences in the C-terminal of RAP-1c [39, 44].

$B$. ovata is considered less pathogenic to cattle than $B$. bovis and B. bigemina, and the similar morphologies and $18 \mathrm{~S}$ RNA gene sequences of $B$. ovata and $B$. bigemina make it difficult to distinguish these two parasites [15]. Further studies are necessary to identify an available marker and to develop a molecular detection assay to differentiate $B$. ovata from B. bigemina.

The ama-1 genes are highly conserved among apicomplexan parasites and have been characterized in several species of Babesia [12]. Several recent studies have used a diagnostic PCR assay based on the ama-1 gene to identify and specifically detect $B$. ovata from questing ticks and cattle [45-47]. The ama-1 gene sequences share an overall identity ranging from 97.8 to $99.6 \%$ at the nucleotide level in a comparison of Chinese isolates with those from other countries [47]. To our knowledge, this is the first report on B. ovata infection in China. B. ovata ama-1 sequences from Henan Province indicated high conservation in three Henan isolates. In contrast to the $B$. bovis and $B$. bigemina parasites in the samples tested, a low prevalence of $B$. ovata in only one province of 14 was observed. A similar result was obtained in a previous study. $B$. ovata infection exhibits lower parasitaemia in infected cattle than $B$. bovis and B. bigemina, thus, it is possible that a low concentration of $B$. ovata DNA exists in field samples that could not be detected by PCR 
[47]. This possibility suggests that additional molecular markers and an adequate number of samples from individual locations of more provinces should be tested.

\section{Conclusions}

We have successfully developed an appropriate and specific nested PCR approach for the detection and discrimination of the important Babesia parasites from different geographical locations in China as well as for epidemiological investigations. B. bovis and B. bigemina were highly prevalent in the sampling areas of the 14 provinces surveyed, but a low presence of $B$. ovata parasites was observed. Further studies concerning the prevalence of B. ovata should be performed to confirm the presence of this parasite in China. Specific serodiagnosis and/or molecular detection based on the rap-1a gene of B. bovis in China will allow for epidemiological surveys.

Moreover, it is the first report on detection and molecular confirmation of B. bovis infection in yaks. Taken together, the data presented in this study suggest that the high sequence heterogeneity in the rap-1a gene from Chinese $B$. bovis isolates might be a great threat to the cattle industry, if RAP-1a protein is used as immunological antigen against Babesia infections in China.

\section{Additional files}

Additional file 1: a. Alignment of partial nucleotide sequences of rap-1a gene from different Chinese isolates. FJ2: $B$. bovis rap- $1 a$ isolate Fujian 2; GS: B. bovis rap-1a isolate Guansu; FJ1: B. bovis rap-1a isolate Fujian 1;GX: B. bovis rap-1a isolate Guangxi; HAN: B. bovis rap-1a isolate Hainan; YN1: $B$. bovis rap-1a isolate Yunnan 1; YN2: $B$. bovis rap-1a isolate Yunnan 2; CQ: B. bovis rap-1a isolate Chongqing; HEN2: $B$. bovis rap-1a isolate Henan 2; HEN 1: B. bovis rap-1a isolate Henan 1; HEN3: B. bovis rap-1a isolate Henan3. Nucleotide substitutions indicated with yellow background. Among these substitutions, nucleotide substitutions affected amino acid modifications indicated with red background. $\mathbf{b}$. Alignment of partial amino acid sequences of rap-1a gene from different Chinese isolates. FJ2: B. bovis RAP-1a isolate Fujian 2; GS: $B$. bovis RAP-1a isolate Guansu; GX: B. bovis RAP-1a isolate Guangxi; CQ: B. bovis RAP-1a isolate Chongqing; HAN: $B$. bovis RAP-1a isolate Hainan; YN1: $B$. bovis RAP-1a isolate Yunnan 1; FJ1: B. bovis RAP-1a isolate Fujian 1; YN2: B. bovis RAP-1a isolate Yunnan 2; HEN2: $B$. bovis RAP-1a isolate Henan 2; HEN 1: B. bovis RAP-1a isolate Henan 1; HEN3: $B$. bovis RAP-1a isolate Henan3. Repeats in RAP-1a gene from different isolates are underlined. (DOC $1086 \mathrm{~kb}$ )

Additional file 2: Variability of $B$. bigemina rap-1c gene. Positions that differ between the different isolates of sequences obtained from gene amplification and cloning for each isolate. (DOC $46 \mathrm{~kb}$ )

\section{Competing interests}

The authors declare that they have no competing interests.

\section{Authors' contributions}

QN, PY, JY and MA carried out the experiments, including PCR, cloning, sequencing and data analysis. QN drafted the manuscript. ZL, GG, GL, JL and HY supervised all work. All authors read and approved the final version of the manuscript.

\section{Acknowledgements}

This study was supported financially by the NSFC (Nos. 31372432, 31201899, 31272556, 31402189, and 31471967); ASTIP; FRIP (2015ZL009), CAAS; Creative
Research Groups of Gansu Province (No. 1210RJIA006); NBCIS CARS-38; Special Fund for Agro-scientific Research in the Public Research (Nos. 201303035 and 201303037), MOA; the 973 Program (2015CB150300), Supporting Program (2013BAD12B03, 2013BAD12B05), MOST, China; and the Jiangsu Co-innovation Center Programme for Prevention and Control of Important Animal Infectious Diseases and Zoonoses, State Key Laboratory of Veterinary Etiological Biology Project. The research was also facilitated by CRP No. 16198/RO IAEA.

\section{Author details}

${ }^{1}$ State Key Laboratory of Veterinary Etiological Biology, Key Laboratory of Veterinary Parasitology of Gansu Province, Lanzhou Veterinary Research Institute, Chinese Academy of Agricultural Science, Xujiaping 1, Lanzhou, Gansu 730046, PR China. ${ }^{2}$ Jiangsu Co-innovation Center for Prevention and Control of Important Animal Infectious Diseases and Zoonoses, Yangzhou 225009, PR China.

Received: 17 August 2015 Accepted: 22 September 2015 Published online: 09 October 2015

\section{References}

1. Lu WS, Yin H, Luo JX, Lu WX, Zhang QC, Dou HF. Discovery of Babesia major in Xinjiang. Symposium of the 3rd Meeting of Chinese Society of Veterinary Parasitology. 1992. p. 220.

2. Yin H, Lu WS, Luo JX. Babesiosis in China. Trop Anim Health Prod. 1997;29:11S-5S.

3. Bai Q, Liu GY. Studies on isolation and preservation of a single species of haemotecytozoon in bovine: isolation and cryopreservation of Babesia ovata in China. Chin J Vet Med. 1990;16:2-4.

4. Luo JX, Yin H, Guan GQ, Yang DY, Liu AH, Ma ML, et al. A comparison of small-subunit ribosomal RNA gene sequences of bovine Babesia species transmitted by Haemaphysalis spp. in China. Parasitol Res. 2005;95:145-9.

5. Luo JX, Yin H, Liu ZJ, Yang DY, Guan GQ, Liu AH, et al. Molecular phylogenetic studies on an unnamed bovine Babesia sp. based on small subunit ribosomal RNA gene sequences. Vet Parasitol. 2005;133:1-6.

6. He L, Feng HH, Zhang WJ, Zhang QL, Fang R, Wang LX, et al. Occurrence of Theileria and Babesia species in water buffalo (Bubalus babalis, Linnaeus, 1758) in the Hubei province. South China Vet Parasitol. 2012;186:490-6.

7. Qi C, Zhou D, Liu J, Cheng Z, Zhang L, Wang L, et al. Detection of Babesia divergens using molecular methods in anemic patients in Shandong Province, China. Parasitol Res. 2011;109:241-5.

8. Slenning BD. Global climate change and implications for disease emergence. Vet Pathol. 2010;47:28-33.

9. Li Y, Luo Y, Cao S, Terkawi MA, Lan DT, Long PT, et al. Molecular and seroepidemiological survey of Babesia bovis and Babesia bigemina infections in cattle and water buffaloes in the central region of Vietnam. Trop Biomed. 2014;31:406-13.

10. Sun CQ, Liu Z, Gao J, Guan G, Ma M, Luo J, et al. Investigations into the natural infection rate of Haemaphysalis qinghaiensis with Piroplasma using a nested PCR. Exp Appl Acarol. 2008;44:107-14.

11. Yokoyama N, Okamura M, Igarashi I. Erythrocyte invasion by Babesia parasites: current advances in the elucidation of the molecular interactions between the protozoan ligands and host receptors in the invasion stage. Vet Parasitol. 2006;138:22-32

12. Chesne-Seck ML, Pizarro JC, Vulliez-Le Normand B, Collins CR, Blackman MJ, Faber BW, et al. Structural comparison of apical membrane antigen 1 orthologues and paralogues in apicomplexan parasites. Mol Biochem Parasitol. 2005;144:55-67.

13. Gaffar FR, Yatsuda AP, Franssen FF, de Vries E. Erythrocyte invasion by Babesia bovis merozoites is inhibited by polyclonal antisera directed against peptides derived from a homologue of Plasmodium falciparum apical membrane antigen 1. Infect Immun. 2004;72:2947-55.

14. Torina A, Agnone A, Sireci G, Mosqueda JJ, Blanda V, Albanese I, et al. Characterization of the apical membrane antigen-1 in Italian strains of Babesia bigemina. Transbound Emerg Dis. 2010;57:52-6.

15. Sivakumar T, Altangerel $\mathrm{K}$, Battsetseg B, Battur B, Aboulaila M, Munkhjargal T, et al. Genetic detection of Babesia bigemina from Mongolian cattle using apical membrane antigen-1 gene-based PCR assay. Vet Parasitol. 2012;187:17-22. 
16. Tonkin ML, Crawford J, Lebrun ML, Boulanger MJ. Babesia divergens and Neospora caninum apical membrane antigen 1 structures reveal selectivity and plasticity in apicomplexan parasite host cell invasion. Protein Sci. 2013;22:114-27.

17. McElwain TF, Perryman LE, Davis WC, McGuire TC. Antibodies define multiple proteins with epitopes exposed on the surface of live Babesia bigemina merozoites. J Immunol. 1987;38:2298-304.

18. Suarez CE, Palmer GH, Jasmer DP, Hines SA, Perryman LE, McElwain TF Characterization of the gene encoding a 60-kilodalton Babesia bovis merozoite protein with conserved and surface exposed epitopes. Mol Biochem Parasitol. 1991;46:45-52.

19. Skuce PJ, Mallon TR, Taylor SM. Molecular cloning of a putative rhoptry associated protein homologue from Babesia divergens. Mol Biochem Parasitol. 1996:77:99-102.

20. Hilpertshauser H, Deplazes P, Meli ML, Hofmann-Lehmann R, Lutz H, Mathis A. Genotyping of Babesia bigemina from cattle from a non-endemic area (Switzerland). Vet Parasitol. 2007;145:59-64

21. Thompson C, Baravalle ME, Valentini B, Mangold A, Torioni de Echaide S, Ruybal $P$, et al. Typification of virulent and low virulence Babesia bigemina clones by 18S rRNA and rap-1c. Exp Parasitol. 2014;141:98-105.

22. Mtshali MS, Mtshali PS. Molecular diagnosis and phylogenetic analysis of Babesia bigemina and Babesia bovis hemoparasites from cattle in South Africa. BMC Vet Res. 2013;9:154.

23. Ybañez AP, Sivakumar $T$, Ybañez RH, Vincoy MR, Tingson JA, Perez ZO, et al. Molecular survey of bovine vector-borne pathogens in Cebu, Philippines. Vet Parasitol. 2013;196:13-20.

24. Sivakumar T, Altangerel K, Battsetseg B, Battur B, Aboulaila M, Munkhjargal T, et al. Genetic characterization of Babesia and Theileria parasites in water buffaloes in Sri Lanka. Vet Parasitol. 2014;200:24-30.

25. Ochirkhuu N, Konnai S, Mingala CN, Okagawa T, Villanueva M, R Pilapil FM, et al. Molecular epidemiological survey and genetic analysis of vector-borne infections of cattle in Luzon Island, the Philippines. Vet Parasitol. 2015. doi:10.1016/j.vetpar.2015.05.019.

26. Tamura K, Stecher G, Peterson D, Filipski A, Kumar S. MEGA6 molecular evolutionary genetics analysis version 6.0. Mol Biol Evol. 2013;30:2725-9.

27. Liu ZL, Zhao JL, Ma LH, Yao BA. Babesia orientalis sp. nov. parasitized in buddalo bubalus babalis in China (piroplasmida: babesiidae). Acta Vet Zootechnica Sin. 1997;28:84-9.

28. Liu J, Yin H, Liu G, Guan G, Ma M, Liu A, et al. Discrimination of Babesia major and Babesia ovata based on ITS1-5.8S-ITS2 region sequences of rRNA gene. Parasitol Res. 2008;102:709-13.

29. Liu A, Guan G, Du P, Gou H, Liu Z, Liu J, et al. Loop-mediated isothermal amplification (LAMP) method based on two species-specific primer sets for the rapid identification of Chinese Babesia bovis and B. bigemina. Parasitol Int. 2012;1:58-63.

30. Liu J, Guan G, Liu A, Li Y, Yin H, Luo J. A PCR method targeting internal transcribed spacers: the simultaneous detection of Babesia bigemina and Babesia bovis in cattle. Acta Parasitol. 2014;59:132-8.

31. Qin SY, Wang $J$, Ning HR, Tan QD, Yin MY, Zhang XX, et al. First report of Babesia bigemina infection in white yaks in China. Acta Trop. 2015;145:52-4.

32. Du XJ, Wang Z, Zhang YT, Liu QS, Han BYC. Seroprevalence of Babesia bigemina and worms in black yaks in Xinjiang Uygur Autonomous Region, north-western China. Anim Husb Vet Med. 2014;1:25 (In chinese).

33. Graves IL, Adams WH, Pyakural S. Recurring hemolytic anemia, babesiasis, and influenza A viruses in a yak at low altitude in Nepal. Am J Vet Res. 1975;36:843-5.

34. Saravanan BC, Das S, Siju SJ, Tewari AK, Sankar M, Kataktalware MA, et al. Babesia bigemina infection in yak (Poephagus grunniens L.): molecular detection and characterization. Vet Parasitol. 2013;194:58-64.

35. Ahanger RR, Godara R, Katoch R, Yadav A, Bhutyal AD, Katoch M, et al. Deltamethrin resistance in field populations of Rhipicephalus (Boophilus) microplus (Acari: Ixodidae) in Jammuand Kashmir, India. Exp Appl Acarol. 2015;67:567-75

36. Teng G, Jiang Z. Economic insect fauna of China. Fasc 39, Acari: Ixodidae. Beijing: Science Press; 1991. p. 81-185. 345-349.

37. Gohil S, Herrmann S, Günther S, Cooke BM. Bovine babesiosis in the $21^{\text {st }}$ century: advances in biology and functional genomics. Int J Parasitol. 2013;43:125-32.

38. Salama AA, Terkawi MA, Kawai S, Aboulaila M, Nayel M, Mousa A, et al. Specific antibody to a conserved region of Babesia apical membrane antigen-1 inhibited the invasion of $B$. bovis into the erythrocyte. Exp Parasitol. 2013;135:623-8.

39. Suarez CE, Palmer GH, Florin-Christensen M, Hines SA, Hötzel I, McElwain TF. Organization, transcription, and expression of rhoptry associated protein genes in the Babesia bigemina rap-1 locus. Mol Biochem Parasitol. 2003;127:101-12.

40. Bhoora R, Quan M, Zweygarth E, Guthrie AJ, Prinsloo SA, Collins NE. Sequence heterogeneity in the gene encoding the rhoptry-associated protein-1 (RAP-1) of Babesia caballi isolates from South Africa. Vet Parasitol. 2010;169:279-88.

41. Mendes TA, Lobo FP, Rodrigues TS, Rodrigues-Luiz GF, daRocha WD, Fujiwara RT, et al. Repeat-enriched proteins are related to host cell invasion and immune evasion in parasitic protozoa. Mol Biol Evol. 2013;30:951-63.

42. Suarez CE, Palmer GH, Hötzel I, McElwain TF. Structure, sequence, and transcriptional analysis of the Babesia bovis rap-1 multigene locus. Mol Biochem Parasitol. 1998;93:215-24.

43. Molad T, Fleiderovitz L, Leibovitz B, Wolkomirsky R, Behar A, Markovics A. Differentiation between Israeli $B$. bovis vaccine strain and field isolates. Vet Parasitol. 2015;208:159-68.

44. Molad T, Erster O, Fleiderovitz L, Roth A, Leibovitz B, Wolkomirsky R, et al. Molecular characterization of the Israeli B. bigemina vaccine strain and field isolates. Vet Parasitol. 2015. doi:10.1016/j.vetpar.2015.06.022.

45. Sivakumar T, Tagawa M, Yoshinari T, Ybanez AP, Igarashi I, Ikehara Y, et al. PCR detection of Babesia ovata from cattle reared in Japan and clinical significance of coinfection with Theileria orientalis. J Clin Microbiol. 2012;50:2111-3.

46. Sivakumar T, Tattiyapong M, Okubo K, Suganuma K, Hayashida K, Igarashi I, et al. PCR detection of Babesia ovata from questing ticks in Japan. Ticks Tick Borne Dis. 2014;5:305-10.

47. Yoshinari T, Sivakumar T, Asada M, Battsetseg B, Huang X, Lan DT, et al. A PCR-based survey of Babesia ovata in cattle from various Asian, African and South American countries. J Vet Med Sci. 2013;75:211-4.

\section{Submit your next manuscript to BioMed Central and take full advantage of:}

- Convenient online submission

- Thorough peer review

- No space constraints or color figure charges

- Immediate publication on acceptance

- Inclusion in PubMed, CAS, Scopus and Google Scholar

- Research which is freely available for redistribution 